Musées, Patrimoine et Culture scientifiques et techniques

$153 \mid 2014$

mai-juin 2014

\title{
Vu du côté des bases : le recueil des données par Trans'Tyfipal $^{\circ}$
}

Jérôme Thomas

\section{(Q) OpenEdition \\ Journals}

Édition électronique

URL : http://journals.openedition.org/ocim/1404

DOI : $10.4000 /$ ocim. 1404

ISSN : 2108-646X

Éditeur

OCIM

Édition imprimée

Date de publication : 25 juin 2014

ISSN : 0994-1908

Référence électronique

Jérôme Thomas, "Vu du côté des bases : le recueil des données par Trans'Tyfipal ${ }^{\circledR}$ », La Lettre de I'OCIM [En ligne], 153 | 2014, mis en ligne le 25 juin 2016, consulté le 30 avril 2019. URL : http:// journals.openedition.org/ocim/1404; DOI : 10.4000/ocim. 1404 


\title{
Vu du côté des bases : le recueil des données par Trans'Tyfipal ${ }^{\circledR}$
}

\author{
Jérôme Thomas \\ Ingénieur chargé de collections muséales, \\ Université de Bourgogne, laboratoire uB-CNRS 6282 Biogéoscience
}

Trans'Tyfipal ${ }^{\circledR}$ est intégré au programme e-ReColNat pour lequel il réalise l'inventaire des spécimens «types » et figurés paléontologiques conservés en France, avec une extension à la zoologie. «Les holotypes, syntypes, lectotypes et néotypes constituent les standards de référence internationaux dont dépend l'objectivité de la nomenclature » (CINZ, 1999, article 72.10, p. 209). Ces « types» et les spécimens figurés à valeur scientifique, mais non nomenclaturale, sont des biens patrimoniaux au sens de l'article Ll du Code du Patrimoine et doivent donc faire l'objet du récolement décennal. Le Code International de Nomenclature Zoologique mentionne que " tout établissement où sont déposés des types devrait : 1) s'assurer que tous sont clairement marqués, de manière à ce qu'ils soient reconnaissables sans ambiguïté ; 2) prendre toute disposition pour leur bonne conservation ; 3) les rendre accessibles pour étude ; 4) publier des listes de types en sa possession ou dont il a la charge et 5) autant que possible,

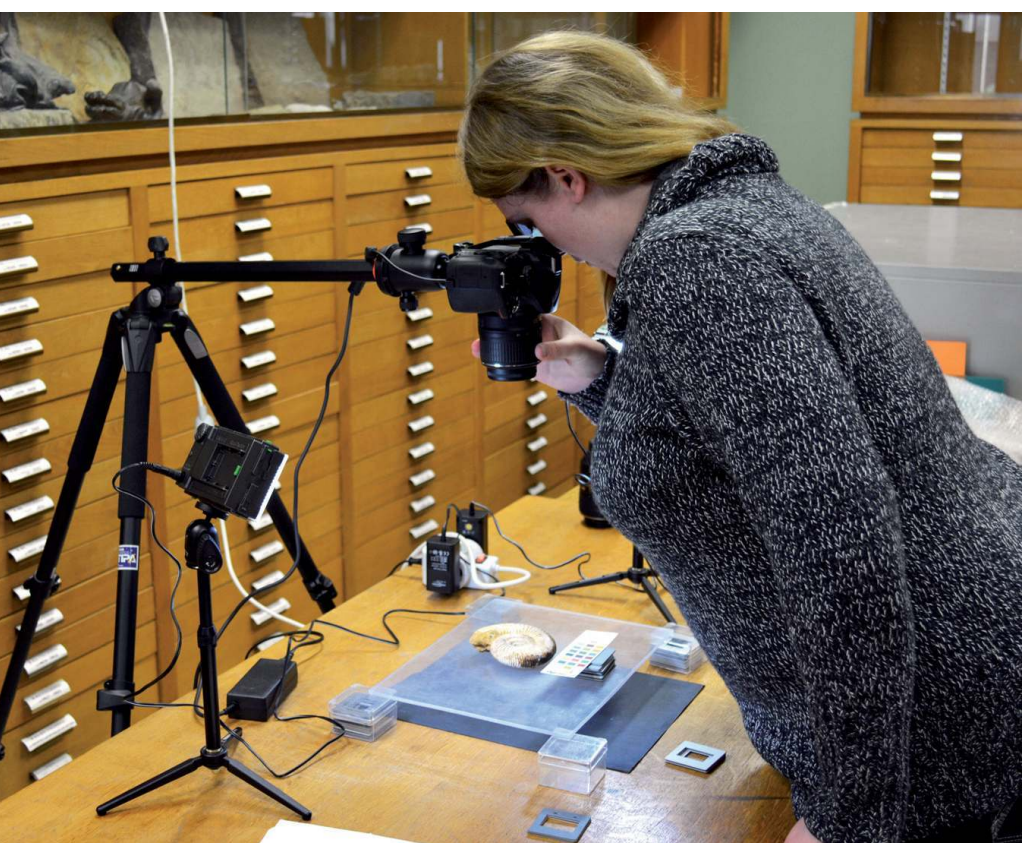

Prise de vue d'un spécimen type (c) Université de Bourgogne/Jérôme Thomas communiquer les renseignements concernant les types " (CINZ, 1999, recommandation 72F. Responsabilité des établissements, p. 210). Le récolement décennal permet donc de transcrire en droit français la recommandation 72F.1., alors que Trans'Tyfipal ${ }^{\circledR}$ est un des outils favorisant les deux dernières mentions (72F.4 et 5).

À ce jour, nous recensons déjà plus de 125000 «types » et figurés paléontologiques pour une trentaine de musées et universités. Ce chiffre est en accroissement régulier en fonction de l'apport des nouvelles publications et des récolements dans les établissements détenteurs, surtout quand on sait que plus de 300 institutions conservent des collections de fossiles.

Dans son analyse des caractéristiques des muséums, Michel Van-Praët recommandait aux muséums de valoriser leurs collections en s'inscrivant dans les démarches globales d'inventaires tels que le portail GBIF, ou via des systèmes spécialisés, comme Trans'Tyfipal ${ }^{\circledR}$ pour les «types » paléontologiques (Van-Praët, 2010, p. 28).

Du point de vue d'un diffuseur comme Trans'Tyfipal ${ }^{\circledR}$ et au moment où s'achève le premier récolement décennal des musées de France, nous avons rencontré trois principaux cas.

1) En raison d'une focalisation du personnel des collections sur le récolement (auquel peut s'ajouter une rénovation de l'établissement et/ou un grand chantier des collections), certaines institutions n'ont pu répondre favorablement à notre sollicitation. Bien que ce constat soit négatif, il s'agit plus d'une indisponibilité temporaire que d'un refus de participer. En effet, ces établissements souhaitent programmer cette valorisation dans les mois à venir.

2) D'autres ont priorisé le récolement des «types » et figurés et ont pu profiter de l'informatisation et de la numérisation de ces spécimens par les équipes de Trans'Tyfipal ${ }^{\circledR}$ sur financement d'e-ReColNat. Ce fut le cas pour les musées de Bourges, Le Mans et Montbéliard, pour lesquels la documentation issue des publications scientifiques et les photographies favorisent le repérage de spécimens et complètent leurs inventaires. 


\section{Points de vue complémentaires}

3) Certains établissements, et plus particulièrement les «musées mixtes » disposant rarement de l'expertise scientifique en sciences naturelles, ont pu profiter de différents projets. Ce fut par exemple le cas du musée municipal de Semur-en-Auxois (21) qui conserve un fonds important de fossiles du Sinémurien (site éponyme de l'étage géologique du Jurassique reconnu internationalement). Un projet réunissant ce musée et le laboratoire uB-CNRS Biogéosciences, avec l'aide technique de Trans'Tyfipal ${ }^{\circledR}$ et un financement de GBIF-France, a permis de documenter, inventorier et numériser l'intégralité de la collection paléontologique (environ 11000 spécimens), et plus particulièrement les 200 spécimens de référence. Ce travail a grandement facilité son récolement.

Dans la majorité des muséums, les récolements devraient avoir permis de mieux documenter les collections et de repérer les spécimens-« types » et figurés. Rappelons que le programme finance l'intervention dans les établissements demandeurs d'équipes d'informatisation et de numérisation des spécimens de référence en paléontologie et zoologie. La fin du récolement en 2014 et la poursuite d'e-ReColNat devraient favoriser ces opérations de valorisation des collections.

\section{Bibliographie}

Commission Internationale de Nomenclature Zoologique. Code International de Nomenclature Zoologique, $4^{e}$ édition, texte français. Londres : The Natural History Museum, Cromwell Road, 1999, 306 p.

Van-Praët, M. Les muséums d'histoire naturelle, lieux d'un dialogue contemporain entre sciences et sociétés, Musées et Collections Publiques de France, $\mathrm{n}^{\circ} 258$ (1), 2010, pp. 24-29.

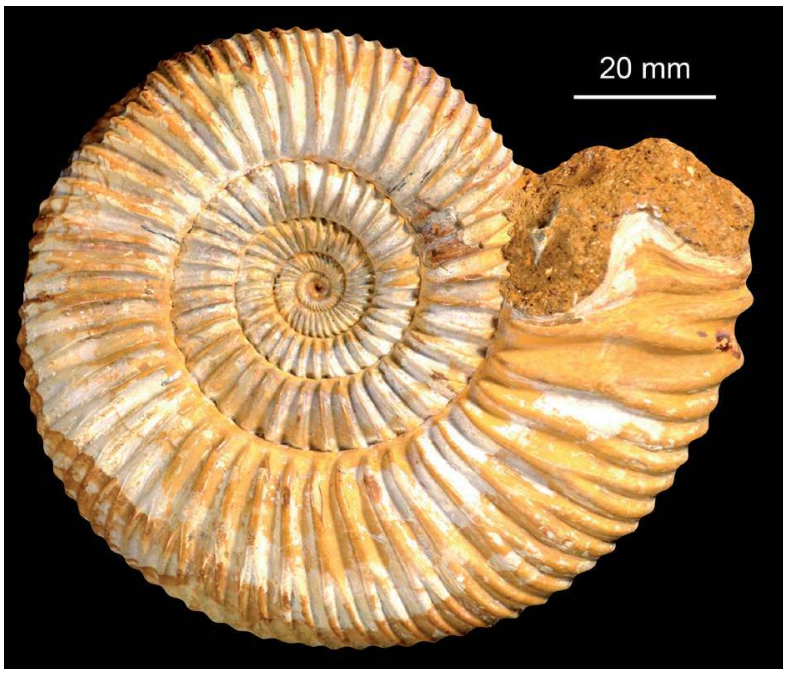

UBGD 10350, holotype de Perisphinctes Besairiei (Collignon, 1959) (c) Université de Bourgogne/Jérôme Thomas 